\title{
Estudio histopatológico de las lesiones hepáticas inducidas por paraquat.
}

Histopathological study of paraquat induced hepatotoxicity.

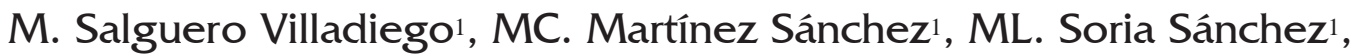
S. García Rodríguez ${ }^{1}$ y G. Repetto Kuhn ${ }^{1}$

\section{RESUMEN}

Se realiza el estudio histopatológico de las lesiones que el paraquat produce en el hígado. El estudio microscópico de los 15 casos revisados indica el posible papel que la lesión del endotelio juega en el desarrollo de la lesión hepatotóxica producida este plaguicida.

Palabras clave: Paraquat, hepatotoxicidad, lesión hepática.

Cuad Med Forense 2005; 11(40):113-117

\section{ABSTRACT}

The aim of this study is to describe the histopathological characteristics of the liver damage in paraquat poisoning. The microscopic characteristics of the liver damage in the 15 cases reviewed emphasize the possible role of the endothelial lesions in the development and pathological expression of liver damage.

Key words: Paraquat, hepatotoxicity, hepatic injury.

Correspondencia: Manuel Salguero. Instituto Nacional de Toxicología y Ciencias Forenses. Avda. Dr. Fedriani, s/n 41009 Sevilla. Tel: 9543712 33. Fax: +34 9543702 62. E-mail: manuel.salguero@mju.es

1 Instituto Nacional de Toxicología y Ciencias Forenses. Departamento de Sevilla. 


\section{INTRODUCCIÓN:}

El paraquat es el plaguicida que más intoxicaciones letales produce en los países desarrollados. Supone una situación clínica extraordinariamente grave con una mortalidad que oscila entre el $50 \%$ y el $78 \%$ [I]. Suele producirse por ingestión con fines suicidas aunque se han descrito casos de intoxicación por vía cutánea en sujetos con lesiones dermoepidérmicas [2].

Tras la ingestión, su absorción a través del tracto digestivo no es muy buena aunque está potenciada por la presencia de emulsificantes y cosolventes en los preparados comerciales. La severa irritación y la necrosis que este compuesto produce en el epitelio gastrointestinal también favorece su paso al torrente circulatorio. Su principal vía de excreción es la renal siendo menos importante la eliminación hepática [3].

La penetración en el interior de la célula se realiza a través del sistema de transporte de poliaminas presente en los neumocitos alveolares y en las células endoteliales. Una vez que este herbicida entra en la célula, sufre una reducción de un electrón formándose el catión paraquat y un anión superóxido reactivo que es convertido en peróxido de hidrógeno por la enzima superóxido dismutasa. Tanto el anión superóxido como el peróxido de hidrógeno reaccionan con los lípidos de las membranas celulares formando hidroperóxidos lipídicos que reaccionan a su vez con otros lípidos de la membrana perpetuando así la acción destructiva [3].

El pulmón es el principal órgano diana de esta sustancia y las lesiones que provoca en este órgano son las más graves siendo las responsables de la severidad del cuadro clínico y la gran mortalidad que produce.

Sin embargo, las publicaciones en relación con la descripción de las lesiones histopatológicas que aparecen en el hígado son mucho menos numerosas y aunque se han establecido a grandes rasgos las principales alteraciones microscópicas [4,5,6,7], la interpretación del desarrollo de las mismas así como sus características histopatológicas no concuerdan en muchos casos con las lesiones que se observan en el hígado de los sujetos que han muerto como consecuencia de la ingestión de paraquat.

\section{MATERIAL Y MÉTODOS:}

Revisamos 15 casos de intoxicación por paraquat recibidos en el Instituto Nacional de Toxicología y Ciencias Forenses de Sevilla. En todos ellos la intoxicación tuvo etiología suicida y se produjo por ingestión del herbicida. Los sujetos murieron en un intervalo de tiempo que oscilaba entre 20 horas y catorce días después de la ingestión. Se estudiaron las lesiones hepáticas de 5 sujetos que fallecieron en los primeros cinco días tras la ingestión, otros cinco cuyo fallecimiento se produjo entre el día 6 y el día 10 después de la ingesta del herbicida y el último grupo de cinco pacientes que murieron entre el día II y el día 14.

Las muestras de hígado fueron talladas e incluidas en parafina y los cortes se tiñeron con hematoxilina-eosina, tinción de plata para reticulina, tricrómico de Masson y PAS-diastasa realizándose posteriormente el estudio histopatológico.

\section{RESULTADOS:}

Dependiendo del tiempo transcurrido entre la ingestión de paraquat y la muerte del paciente, pueden diferenciarse tres etapas en el desarrollo de las lesiones:

1) Lesiones iniciales con degeneración hidrópica de los hepatocitos pericentrales: aparecen en las primeras 48 horas después de la ingestión. 
2) Necrosis de los hepatocitos pericentrales con hemorragia: puede apreciarse a partir del segundo día y persiste aún el día catorce.

3) Lesión colangiodestructiva con cuadro colestásico que comienza entre el $5^{\circ}$ y $6^{\circ}$ día y se agrava a medida que el tiempo de supervivencia tras la intoxicación aumenta.

Lesiones iniciales.- Se observa una acusada tumefacción de los hepatocitos pericentrales (Figura I) que es constante en todos los lobulillos. Las células están muy aumentadas de tamaño; el citoplasma apenas se tiñe y adquiere un aspecto apolillado.

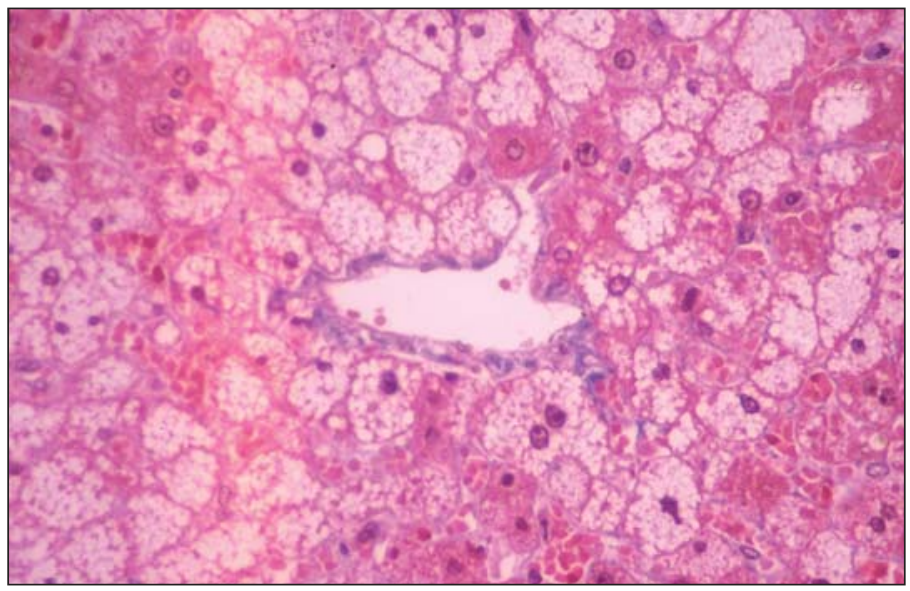

Figura 1: Degeneración hidrópica de los hepatocitos pericentrales.

Necrosis de los hepatocitos pericentrales con hemorragia.- A partir del segundo día, la lesión irreversible que sufren los hepatocitos se manifiesta por la necrosis de los mismos que se acompaña de una intensa congestión sinusoidal, necrosis de las células endoteliales y focos hemorrágicos. Los hepatocitos preservados en torno a los focos de necrosis muestran una acusada esteatosis microvesicular.

En algunos casos la necrosis es más extensa y afecta también a los hepatocitos mediozonales quedando preservados solamente los hepatocitos periportales.

Lesión colangiodestructiva con cuadro colestásico.- Después del quinto día comienzan a observarse las lesiones colestásicas con necrosis de las células epiteliales de los ductos biliares, desprendimiento de las mismas al interior de las luces de los conductillos y atipia regenerativa de las células del epitelio biliar en días posteriores (Figuras 2 y 3). Este cuadro colangiodestructivo es más llamativo a medida que aumenta el tiempo transcurrido desde la ingestión de paraquat.

En la pared de algunos ductos puede advertirse además un infiltrado inflamatorio mixto constituído por linfocitos y leucocitos polimorfonucleares neutrófilos (colangitis).

\section{DISCUSIÓN:}

El paraquat es un compuesto con capacidad hepatotóxica intrínseca que produce una lesión hepatocanalicular. Los estudios histopatológicos coinciden en que aparece una lesión precoz que consiste en la necrosis de la zona 3 del lobulillo y otra más tardía que es la colestasis con lesión de los conductillos biliares $[8,9]$. 


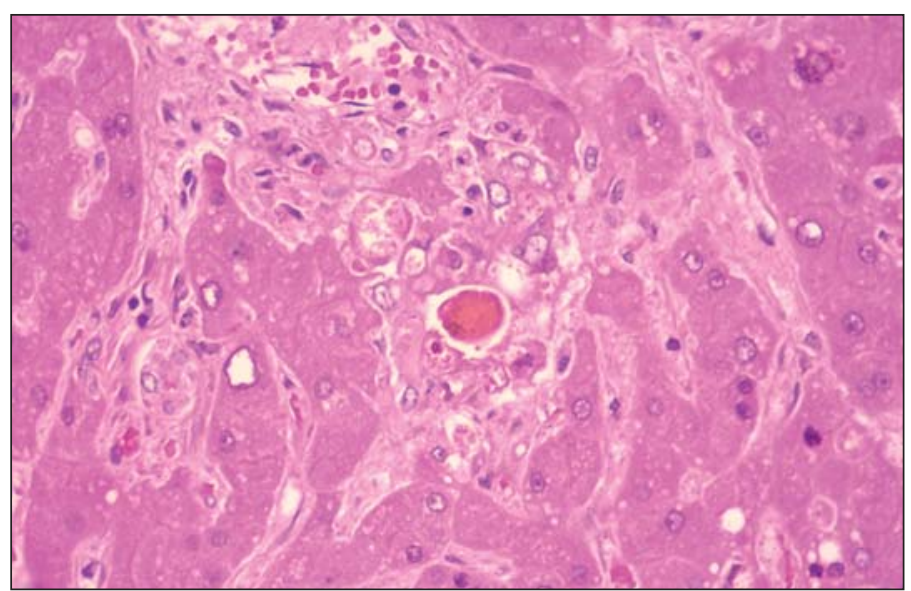

Figura 2: Cuadro colestásico con moldes de bilis en los conductillos.

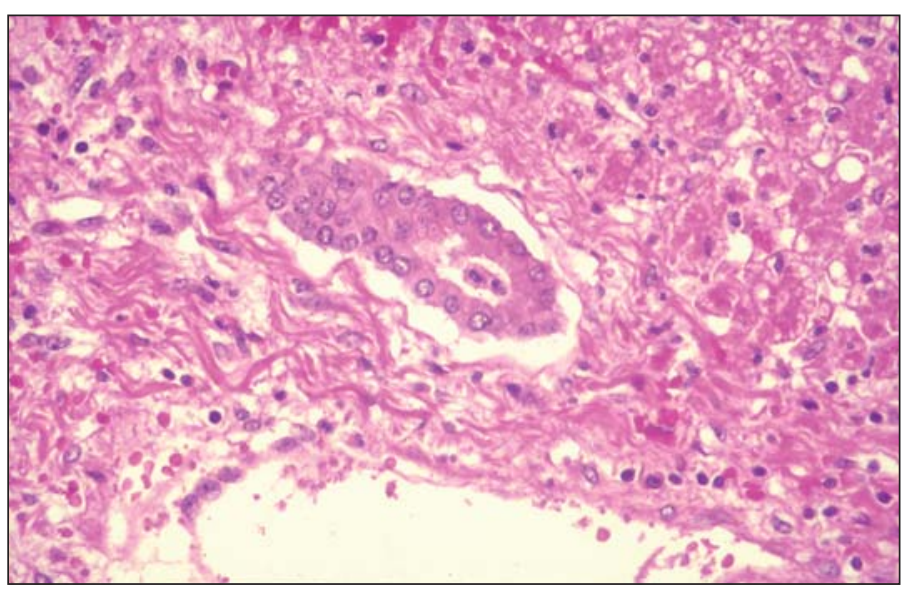

Figura 3: Atipia regenerativa del epitelio biliar.

Algunos autores cuestionan la necrosis hepatocelular y piensan que puede ser explicada por la situación de choque que precede al fallecimiento de los sujetos intoxicados [8]. Sin embargo, la tumefacción de los hepatocitos pericentrales que aparece en las primeras horas y que posteriormente desemboca en la necrosis de la zona 3 del lobulillo, refleja la capacidad hepatotóxica intrínseca del paraquat. Además, las características microscópicas de esta necrosis indican que no se trata de una simple lesión hipóxica-isquémica provocada por el choque.

La lesión más referida del paraquat, y considerada como la más característica, es la colestasis que suele comenzar entre los días tercero y sexto después de la ingestión y permanece durante largo tiempo habiéndose descrito en sujetos que han fallecido 70 días más tarde [4]. En casos de intoxicación por exposición dérmica en pacientes con lesiones cutáneas, llega a convertirse en un cuadro colestásico crónico [3].

En ningún momento se ha descrito el probable papel que la lesión de las células endoteliales de los sinusoides puede jugar en el desarrollo de las lesiones tanto de los hepatocitos como de las células del epitelio biliar en las intoxicaciones por paraquat. 
El componente hemorrágico observado en la necrosis pericentral probablemente sea el reflejo de una lesión de las células endoteliales de los sinusoides y en las lesiones colangiodestructivas, la lesión del endotelio de la microcirculación del árbol biliar intrahepático puede contribuir de forma definitiva en el desarrollo de las lesiones colestásicas. Además esta microcirculación puede ser la principal vía de acceso del herbicida a las células del epitelio biliar, ya que la excreción biliar del paraquat es mínima y no se justifica la gravedad de las lesiones observadas con ese posible contacto directo del plaguicida excretado por la bilis con los colangiocitos que tapizan los conductillos como han preconizado algunos autores [ 10$]$.

La microcirculación del sistema biliar ductal intrahepático la proporciona el plexo capilar peribiliar que deriva exclusivamente de la arteria hepática lo que le confiere a los conductillos biliares una mayor susceptibilidad a la isquemia.

Por lo tanto, creemos que además de la lesión de los hepatocitos y de las células del epitelio biliar, la lesión de las células endoteliares de los sinusoides juega un papel esencial en la génesis de las lesiones inducidas por el paraquat en el hígado. Este daño endotelial explica la morfología de las lesiones microscópicas observadas.

Las células endoteliales constituyen el $48 \%$ de las células que se encuentran en los sinusoides y cada vez reciben más atención en el estudio de las lesiones hepatotóxicas [ I I]. Todo parece indicar que estas células juegan un importante papel en las respuestas tóxicas del hígado. Actualmente se consideran como reacciones complejas en las que cada uno de los tipos celulares que componen este órgano interactúa con los otros condicionando tanto la fisiopatología como las lesiones histopatológicas que aparecen tras la acción de un tóxico. El daño hepático inducido por el paraquat es un ejemplo muy demostrativo de esta interacción.

\section{AGRADECIMIENTOS:}

A D D $^{a}$ Ángeles Correa del Valle y Da Marisa Vázquez Pereira, por el procesado y tinción de las muestras histopatológicas.

\section{BIBLIOGRAFÍA:}

I.- Nogué S, Dueñas A. Intoxicación por paraquat: un puzzle al que le faltan piezas. Med Clin. 2000; II5: 546-548

2.- Bataller R, Bragulat E, Nogue S. Gorbig MN, Bruguera M. Rodes J. Prolonged cholestasis after acute paraquat poisoning through skin absorption. Am J Gastroenterol. 2000;95:I340-1343

3.- Ecobichon D.(200I) Toxic effects of pesticides pp.763-810 En: Casarett and Doull's Toxicology. The basic science of poisons 6th edition Ed. Klaasen C.D. McGraw Hill. New York.

4.- Mullick FG, Ishak KG, Mahabir R, Stromeyer FW. Hepatic injury associated with paraquat toxicity in humans. Liver 1981; I: 209-22I 5.- Parkinson C. The changing pattern of paraquat toxicity in man. Histopathology 1980; 4: 171-183

6.- Takegoshi K, Nakamura Y, Ohta M, Thoyama T, Okuda K, Kono $\mathrm{N}$. Ligth and eletron microscopic study of the liver in paraquat poisoning. Liver 1988; 8: 330-336

7.- Vadnay I. Hepatic alterations in experimental paraquat intoxicacion. Ex. Toxicol. Pathol. 1992; 45:355-364

8.- Zimmerman H J (1999) Hepatotoxicity. The adverse effects of drugs and other chemicals on the liver. Second edittion. Lippincott Williams and Wilkins. Philadelphia.

9.- Zimmerman H J, Ishak K G (2002) Hepatic injury due to drugs and toxins pp 621-709. En: MacSween R N M, Burt A D, Portman B C, Ishak K G, Scheuer P.J. Anthony P P (2002) Pathology of the liver. Fourth edition. Churchill-Livingstone, London

10.- Oneyama H P, Oehme F.W. A literature review of paraquat toxicity. Vet Hum Toxicol 1984; 26: 494-502

II.- Laskin D L. Sinusoidal lining cells and hepatotoxicity. Toxicol Pathol 1996; 24: II2-118 